ORIGINAL ARTICLE

\title{
Melanoma inhibitor of apoptosis protein is expressed differentially in melanoma and melanocytic naevus, but similarly in primary and metastatic melanomas
}

\author{
J Gong, N Chen, Q Zhou, B Yang, Y Wang, X Wang
}

J Clin Pathol 2005;58:1081-1085. doi: 10.1136/jcp.2005.025817

See end of article for authors' affiliations

Correspondence to:

$\operatorname{Dr} Q$ Zhou, Pathology

Department, West China

Hospital, West China

Medical School, Sichuan

University, Chengdu

610041, China;

zhouqiao@mcwcums.com

Accepted for publication 2 March 2005
Background: Malignant melanoma is highly resistant to current treatments. The inhibitor of apoptosis protein (IAP) family member, melanoma IAP (ML-IAP), is overexpressed in some melanoma cell lines, rendering them resistant to apoptotic signals. Targeting ML-IAP is a promising approach to treating melanoma. However, the status of ML-IAP expression in human melanoma tissues and the difference in expression between melanoma and melanocytic naevus are not known.

Aims: To investigate these issues.

Methods: ML-IAP expression in 48 archived patient samples (34 melanomas and 14 dermal naevi) was assessed by immunohistochemistry and by in situ hybridisation and reverse transcription polymerase chain reaction (RT-PCR) assays developed for the study.

Results: Expression of ML-IAP was detected in 47.6-70.6\% (10 of 21 to 24 of 34) of the melanomas, varying with detection methods. The expression rate in melanoma was much higher than that in melanocytic naevus (10.0-21.4\%; one of 10 to three of 14). No significant difference was seen between primary and secondary melanomas. ML-IAP expression rates assessed by the three methods were in agreement.

Conclusions: The ML-IAP expression rate in archived melanoma tissues is around $50-70 \%$, with no difference between primary and secondary melanomas. A small number of dermal naevi ( 20\%) also expressed ML-IAP.
M alignant melanoma is highly aggressive with a poor response to current treatments. ${ }^{1}$ Melanoma inhibitor of apoptosis protein (ML-IAP) is a recently identified member of the antiapoptotic IAP family characterised by the baculoviral inhibitory repeat (BIR) domain(s)..$^{2-6}$ ML-IAP is overexpressed in some melanoma cell lines, rendering them resistant to apoptotic signals. ${ }^{2}{ }^{3}$ SMAC (second mitochondrial activator of caspases), a proapoptotic molecule released from mitochondria, can bind to and antagonise ML-IAP. ${ }^{7-11}$ Thus, immunotherapy ${ }^{12-14}$ and SMAC based peptides that target ML-IAP $^{15}{ }^{16}$ are promising new therapeutic approaches to melanoma treatment.

\section{"The overall status of melanoma inhibitor of apoptosis protein expression in human melanoma tissues is unknown"}

The successful application of these novel treatments requires ML-IAP expression to be assessed in patients. However, the overall status of ML-IAP expression in human melanoma tissues is unknown. Moreover, differential expression of ML-IAP (if any) in benign and malignant melanocytic lesions and in primary and secondary melanomas may indicate its roles in the development and progression of malignant melanoma. This important question has not been studied either.

To address these issues, we used immunohistochemistry and developed in situ hybridisation and reverse transcription polymerase chain reaction (RT-PCR) assays to evaluate MLIAP expression in 48 archived patient samples (34 melanomas and 14 dermal naevi). We detected ML-IAP in 47.6$70.6 \%$ ( 10 of 21 to 24 of 34 ) of the melanomas by various methods, but only in $10.0-21.4 \%$ (one of 10 to three of 14) melanocytic naevi $(\mathrm{p}<0.05)$. In melanocytic naevi, ML-IAP was characteristically seen in the type A epithelioid naevus cells. Importantly, we found no differences in the expression of ML-IAP between primary invasive melanomas and secondary melanomas. The expression rates assessed by the three methods were in general agreement. Our present study is the first to evaluate ML-IAP expression in human melanocytic lesional tissues. These findings are useful for understanding ML-IAP function in melanocytic lesions and for applying potential treatments targeting ML-IAP.

\section{MATERIALS AND METHODS}

\section{Tissue samples and primary melanocyte}

All cases and tissue samples were from the authors' institution. Consecutive cases were screened for tissue adequacy for our study, and 48 cases were used, namely: 19 primary cutaneous and mucosal melanomas (patient ages, 20-83 years; mean, 53.6, male to female ratio, $12: 7), 15$ metastatic melanomas (patient ages, 24-80 years; mean, 49.9; male to female ratio, 9:6), and 14 dermal naevi (patient ages, 1-58 years; mean, 25.5; male to female ratio $4: 10)$. All cases were reviewed and immunostained with melanoma associated markers MART-1, HMB-45, and S- 100. The primary cutaneous melanomas included were of Clark's level II or above. Metastases were mainly to lymph nodes and bones.

The human neonatal primary melanocyte cell line HEMnMP was purchased from Cascade Biologics Inc (Portland, Oregon, USA).

Abbreviations: $\mathrm{BIR}$, baculoviral inhibitory repeat; DIG, digoxigenin; $M L-I A P$, melanoma inhibitor of apoptosis protein; RT-PCR, reverse transcription polymerase chain reaction; SMAC, second mitochondrial activator of caspases; SSC, saline sodium citrate 

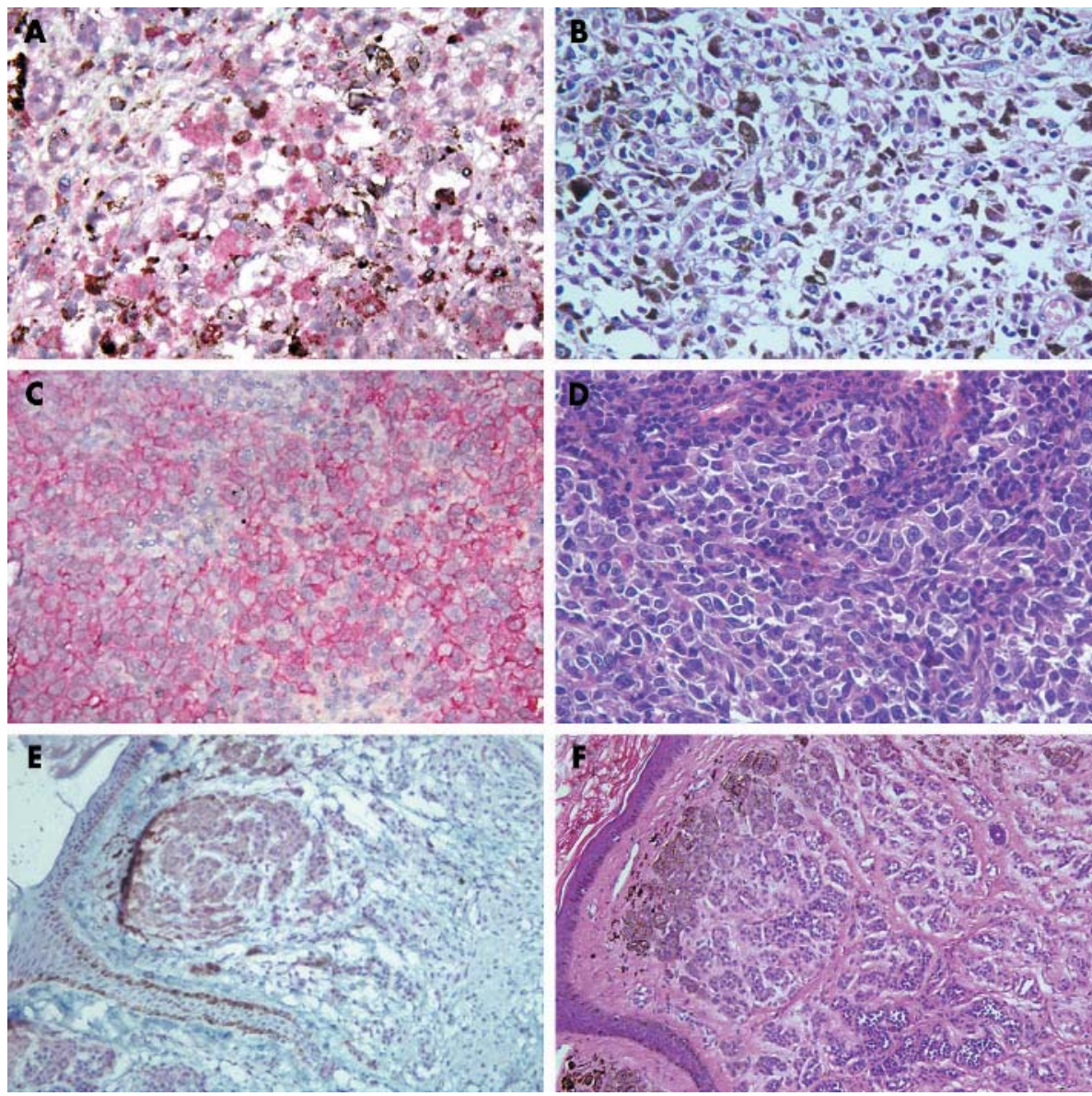

Figure 1 Immunohistochemical detection of melanoma inhibitor of apoptosis protein (ML-IAP) in tissue sections. (A, C, E) Streptavidin-biotin alkaline phosphatase immunostaining with anti-ML-IAP antibody in formalin fixed, paraffin wax embedded tissue sections; $(B, D, F)$ the corresponding haematoxylin and eosin histology. AP red was used for visualisation of immunostaining, with the red colour of the signal contrasting well with the melanin. (A, B) ML-IAP immunoreactivity seen as $(A)$ red cytoplasmic precipitates in (B) a pigmented melanoma. (C, D) Positive ML-IAP staining seen as (C) cytoplasmic rims around nuclei in (D) closely packed melanoma cells of a non-pigmented melanoma. (E, F) Moderate ML-IAP positivity in (E) superficial epithelioid naevus cells in (F) a dermal naevus. (A$D)$ : original magnification, $\times 400 ;(E$, F): original magnification, $\times 100$.

\section{Immunohistochemistry}

The following primary antibodies were used: rabbit antihuman livin (ML-IAP) polyclonal antibody (1/500 dilution; R\&D Systems, Minneapolis, Minnesota, USA), mouse antihuman HBM45 monoclonal antibody (1/50 dilution; Dako, Glostrup, Denmark), mouse antihuman melan-A (MART-1) monoclonal antibody ( $1 / 50$ dilution; Dako), and rabbit antihuman S100 polyclonal antibody (1/200 dilution; Zymed, San Francisco, California, USA). Biotin labelled goat antirabbit or antimouse IgG and alkaline phosphatase coupled streptavidin were from Zymed. Antigen retrieval was by high pressure boiling in $0.01 \mathrm{M}$ citrate buffer $(\mathrm{pH} 6.0)$ for three minutes. Standard streptavidin-biotin alkaline phosphatase immunostaining was applied. The AP red detection kit (Zymed) was used for visualisation and Mayer's haematoxylin was used as the counterstain. Omission of the primary antibody was used as the negative control. Positivity was defined either as diffuse positive

Table 1 ML-IAP immunohistochemistry

\begin{tabular}{llll}
\hline & N & Negative (\%) & Positive (\%) \\
\hline Primary MM & 19 & $5(26.3)$ & $14(73.7)$ \\
Metastatic MM & 15 & $5(33.3)$ & $10(66.7)$ \\
Dermal naevus & 14 & $11(78.6)$ & $3(21.4)$ \\
\hline
\end{tabular}

ML-IAP, melanoma inhibitor of apoptosis protein; MM, malignant melanoma.

Fisher's exact test: primary versus metastatic $M M, p=0.7176$; primary $M M$ versus dermal naevus, $p=0.0049$; metastatic $M M$ versus dermal naevus, $p=0.0253 ; M M$ (primary and metastatic) versus dermal naevus, $p=0.0033$ reactivity or strong reactivity in more than $5 \%$ of tumour cells.

\section{In situ hybridisation}

ML-IAP oligonucleotide probes were designed according to the cDNA sequence (GenBank XM_012922) as follows and were synthesised by Gibco (Gaithersburg, Maryland, USA): 5'-CAAAGACGATGGACACGGC-3' (antisense) and 5' GCCGTGTCCATCGTCTTTG-3' (sense). The digoxigenin (DIG) oligonucleotide Tailing Kit (Roche, Germany) was used to label the probes according to the procedures recommended by the manufacturer. Labelling efficiency and optimal dilutions were determined by dot blot of serially diluted probes.

Chromogenic in situ hybridisation and detection using the DIG nucleic acid detection kit (Roche) was carried out according to protocols described previously. ${ }^{17}$ Briefly, $4 \mu \mathrm{m}$ sections were treated with $30 \mu \mathrm{g} / \mathrm{ml}$ proteinase $\mathrm{K}$ for 20 minutes at $37^{\circ} \mathrm{C}$. Hybridisation was performed for 16 hours at $37^{\circ} \mathrm{C}$, and the slides were then washed sequentially with $2 \times$ saline sodium citrate (SSC), $1 \times$ SSC, and $0.5 \times$ SSC. After blocking, the sections were incubated with anti-DIG-AP (1/ 500 dilution; Roche) at $37^{\circ} \mathrm{C}$ for two hours. Detection was by incubating with NBT/BCIP solution $(1 / 50)$ at $37^{\circ} \mathrm{C}$ in the dark for three to five hours. Slides were counterstained with $1 \%$ methyl green. Positive signals were deep blue cytoplasmic precipitates.

\section{RT-PCR}

Primers for ML-IAP were designed as follows and synthesised by Genebase (Shanghai, China): 5'-ATGGGCTCTGAGGAGTTGCGTC-3' (upstream) and 5'-CATAGCAGAAGAAGC- 


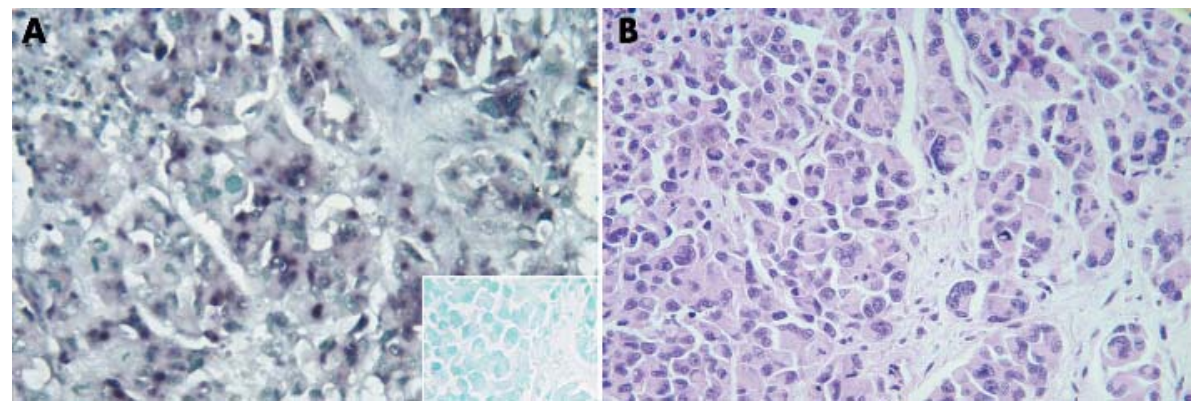

Figure 2 In situ hybridisation using digoxigenin labelled oligonucleotide probes for melanoma inhibitor of apoptosis protein in tissue sections. NBT/ $B C I P$ was used for visualisation and methyl green for counterstaining. (A) Cytoplasmic, deep blue positive signals in a malignant melanoma detected with the antisense probe. Inset: Lack of signal when the control sense probe was used. (B) Corresponding haematoxylin and eosin histology of the same case. Original magnification, $\times 400$.

ACCTCACCTTG-3' (downstream). The housekeeping $\beta$ actin gene was used as an internal control and was amplified using the following primers $^{18}$ : 5'-TGGAGAAAATCTGGCACCAC-3' (upstream) and 5'-GAGGCGTACAGGGATAGCAC-3' (downstream). Primers were designed so that the amplification regions span introns to avoid false positivity caused by genomic DNA contamination.

Total RNA from formalin fixed or formalin fixed and paraffin wax embedded tissues and the primary melanocyte HEMn-MP cell line was isolated with the Trizol reagent (Invitrogen, Carlsbad, California, USA), as recommended by the manufacturer. For paraffin wax embedded tissues, sections $(10 \mu \mathrm{m})$ were dewaxed and digested with proteinase $\mathrm{K}(1 \mathrm{mg} / \mathrm{ml})$ at $55^{\circ} \mathrm{C}$ for 10 hours before RNA isolation. Reverse transcription was carried out in a $20 \mu \mathrm{l}$ mixture containing $5 \mu \mathrm{g}$ total RNA, $0.5 \mu \mathrm{g}$ oligo(dT) 18 primer, $2 \mu \mathrm{l}$ of $10 \mathrm{mmol} /$ litre dNTP, $0.1 \mu \mathrm{l} \mathrm{of} 1 \mathrm{~mol} /$ litre dithiothreitol, and $1 \mu \mathrm{l}$ of M-Mulv reverse transcriptase (Fermentas, Hanover, Maryland, USA), for 60 minutes at $42^{\circ} \mathrm{C}$, followed by 10 minutes at $72^{\circ} \mathrm{C}$.

PCR was carried out with Taq DNA polymerase (Takara, Japan). An initial denaturation at $95^{\circ} \mathrm{C}$ for three minutes, was followed by 30 cycles of 30 seconds at $95^{\circ} \mathrm{C}, 30$ seconds at $58^{\circ} \mathrm{C}$, and 35 seconds at $72^{\circ} \mathrm{C}$, then a final extension at $72^{\circ} \mathrm{C}$ for 10 minutes. PCR products were resolved on a $2 \%$ agarose gel and visualised by ethidium bromide staining.

\section{STATISTICAL ANALYSIS}

The Statistica 5.0 (StatSoft Inc, Tulsa, Oklahoma, USA) package was used for statistical analysis.

\section{RESULTS}

ML-IAP protein expression differs in melanomas and naevi, but is similar in primary and metastatic melanomas

As shown in table 1, ML-IAP protein was detected immunohistochemically in most of the primary (14 of 19) and metastatic (10 of 15) melanomas, with moderate to

Table 2 In situ hybridisation for ML-IAP

\begin{tabular}{llll}
\hline & N & Negative (\%) & Positive (\%) \\
\hline Primary MM & 13 & $5(38.5)$ & $8(61.5)$ \\
Metastatic MM & 15 & $5(33.3)$ & $10(66.7)$ \\
Dermal naevus & 10 & $9(90.0)$ & $1(10.0)$ \\
\hline
\end{tabular}

ML-IAP, melanoma inhibitor of apoptosis protein; MM, malignant melanoma.

Fisher's exact test: primary versus metastatic $M M, p=1.0000$; primary $M M$ versus dermal naevus, $p=0.0288$; metastatic $M M$ versus dermal naevus, $\mathrm{p}=0.0119 ; \mathrm{MM}$ (primary and metastatic) versus dermal naevus, $\mathrm{p}=0.0078$ strong reactivity (fig $1 \mathrm{~A}-\mathrm{D}$ ). Interestingly, three of the 14 dermal naevi were also positive, although weaker, mainly within the type A epithelioid naevus cells (fig lE, F). Type B and $\mathrm{C}$ naevus cells were less reactive, as were other naevus samples. The difference between melanomas and naevi, but not between primary and metastatic melanomas, was significant.

The ML-IAP mRNA expression rate differs between melanoma and naevus, but is similar between primary and metastatic melanomas, as assessed by in situ hybridisation

In situ hybridisation was successful on 28 melanomas (fig 2) and 10 naevi. The results as summarised in table 2 showed significantly higher positivity for ML-IAP in primary (eight of 13) and metastatic melanomas (10 of 15) than in naevi (one of 10). Again, no significant difference was seen between the primary and the metastatic melanomas.

\section{ML-IAP mRNA in melanoma tissues analysed by RT- PCR}

RT-PCR results were obtained from 21 melanoma samples (18 primary and three metastatic), and the ML-IAP product was detected in 10 cases. Eight of 18 primary and two of three metastatic melanomas were positive (Fisher's exact test of primary versus metastatic, $p=0.9985$ ). Figure 3 shows the typical results of six primary and two metastatic melanoma samples compared with the primary melanocyte HEMn-MP cell line (in which ML-IAP was not detected). Variations in the amount of ML-IAP amplification product relative to $\beta$ actin indicated that ML-IAP mRNA values vary among melanomas, and quantitative assays would be required for further analysis.

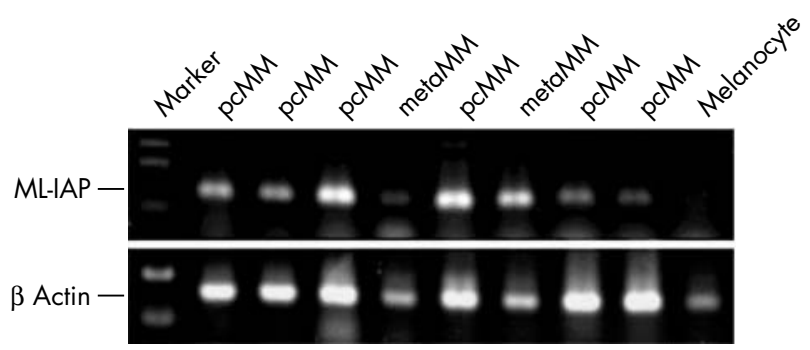

Figure 3 Detecting melanoma inhibitor of apoptosis protein (ML-IAP) in melanoma tissue samples by reverse transcription polymerase chain reaction. Results from six primary cutaneous ( $\mathrm{pc} M \mathrm{MM}$ ) and two metastatic (metaMM) melanomas are shown and compared with the primary melanocyte HEMn-MP cell line. The upper panel is for the $130 \mathrm{bp}$ product of ML-IAP, whereas the lower panel is for the $190 \mathrm{bp}$ product of $\beta$ actin as internal control. 
Table 3 Method comparison for ML-IAP detection in melanoma

\begin{tabular}{llll}
\hline & N & Negative (\%) & Positive (\%) \\
\hline IHC & 34 & $10(29.4)$ & $24(70.6)$ \\
ISH & 28 & $10(35.7)$ & $18(64.3)$ \\
RT-PCR & 21 & $11(52.4)$ & $10(47.6)$ \\
\hline
\end{tabular}

IHC, immunohistochemistry; ISH: in situ hybridisation; ML-IAP, melanoma inhibitor of apoptosis protein; RT-PCR, reverse transcription polymerase chain reaction.

IHC versus ISH, $p=0.5937$; IHC versus RT-PCR, $p=0.1523 ;$ ISH versus RT-PCR, $\mathrm{p}=0.2625$.

Comparison of the three methods for ML-IAP detection Table 3 summarises ML-IAP expression in melanomas assessed by the three methods. Although apparently different, statistical analysis showed no significant differences between the methods, indicating that all three methods were comparable in overall performance. The lower ML-IAP positive rate seen in the RT-PCR assay may be attributable to difficulties in retrieving intact ML-IAP mRNA from formalin fixed and paraffin wax embedded tissues for successful amplification. It is possible that fresh tissue samples would yield higher positive rates.

\section{Correlation of ML-IAP expression with clinicopathological variables}

Spearman rank correlation analysis of ML-IAP expression with melanoma differentiation markers HMB-45, MART-1, and S-100 and common clinical parameters showed only a weak correlation between ML-IAP and HMB45 $\left(r_{\mathrm{s}}=0.36\right.$; $\mathrm{p}=0.0159)$, and between ML-IAP and patient age $\left(r_{\mathrm{s}}=0.39 ; \mathrm{p}=0.0056\right)$. The melanoma cases were mostly of advanced stage, which did not allow a meaningful survival analysis.

\section{DISCUSSION}

ML-IAP belongs to the IAP family of proteins that characteristically contain one or more BIR domain. ${ }^{2-6}$ ML-IAP may directly inhibit caspases in a BIR dependent manner, ${ }^{23}$ although recent data indicate that it prevents cell death mainly by sequestering SMAC. ${ }^{19}$ Although named melanoma IAP because of overexpression in some melanoma cell lines, ${ }^{23}$ ML-IAP is expressed in other tumours and in some fetal tissues. $^{2}$

Malignant melanoma is highly aggressive and resistant to current anticancer treatments, particularly chemotherapy that may kill tumour cells by inducing apoptosis. ${ }^{2}$ Several groups have reported the potential use of novel approaches targeting ML-IAP. ${ }^{12-14}$ For example, Schmollinger et al used irradiated tumour cells as vaccines, and found high titre antiML-IAP antibodies and ML-IAP specific CD4+/CD8+ $\mathrm{T}$ cells. ${ }^{12}{ }^{13}$ Andersen et al found that melanoma infiltrating $\mathrm{T}$ cells may recognise ML-IAP derived peptides, and T cells isolated by magnetic beads coated with ML-IAP peptide/HLAA2 complex may exert cytotoxic effects on HLA matched melanoma cells. ${ }^{14}$ Because the mitochondrial protein SMAC, which is released during intrinsic apoptotic responses, ${ }^{7-11}$ binds to and antagonises ML-IAP," ${ }^{11}$ high affinity peptides based on the SMAC amino acid sequence may serve as potential anti-melanoma drugs. ${ }^{15}{ }^{16}$ To apply these novel approaches clinically, it is necessary to assess the ML-IAP expression status in patients, as is the case for Herceptin treatment of breast cancer, which requires HER-2 gene expression to be assessed in tumour tissues. ${ }^{20}$ Lack of HER-2 overexpression in breast cancer or other tumours (for example, non-Hodgkin lymphoma ${ }^{21}$ ) indicates that antiHER-2 treatment will not be successful.
The expression status of ML-IAP in human melanoma and benign melanocytic lesion tissues has not been evaluated, although Nachmias et al used a monoclonal antibody and western blotting to examine ML-IAP in primary cell cultures derived from melanomas, with 10 of 27 samples being positive. ${ }^{22}$ In our present study, we found overall ML-IAP positive rates between $47.6 \%$ ( 10 of 21 ) and $70.6 \%$ ( 24 of 34 ) in the melanomas. The variations in positivity could result from differences in materials (tissue versus cell culture), antibodies (polyclonal versus monoclonal), and methods (immunohistochemistry etc versus western blot). As with HER-2 assays, ${ }^{23}$ further testing and standardisation are needed for ML-IAP to be assayed quantitatively in patients. Testing different sample types (particularly fresh frozen tissues) and the optimisation of assay methods will be of great value.

\section{"Whether patients bearing melanoma inhibitor of apop- tosis protein expressing naevi are at greater risk for developing melanoma is worthy of further study"}

Interestingly, we also found that about $20 \%$ of dermal naevi showed ML-IAP positivity, mainly in the superficial, type A epithelioid naevus cells. The ML-IAP positive patients were 26-40 years old, but none of the younger (ages, 1-9) or older patients showed positivity. Another IAP family member, survivin, was also reported to be present in benign melanocytic lesions. ${ }^{24}$ Whether patients bearing these IAP expressing naevi are at greater risk for developing melanoma is worthy of further study. It would also be interesting to investigate whether dysplastic naevi, recognised precursors of melanoma, ${ }^{25} 26$ show higher expression of ML-IAP than common dermal naevi.

It was reported that the metastatic phenotype may be associated with apoptotic dysfunction, ${ }^{27}$ and enhanced resistance to apoptosis in metastatic melanoma cells was associated with inactivation of another key proapoptotic molecule, Apaf-1. ${ }^{28}$ We found no significant differences in ML-IAP expression between primary invasive and metastatic melanomas, indicating that ML-IAP probably did not contribute to the development of metastatic potential or to changes in apoptosis resistance in metastatic melanomas. However, the possibility that a difference exists between noninvasive melanoma (melanoma in situ, Clark's level I) and invasive melanoma (Clark's levels II-V) cannot be excluded.

\section{ACKNOWLEDGEMENTS}

This work was supported in part by grants from the Natural Science Funding Committee (NSFC, 30125023) and Ministry of Science and Technology (2002CCA01400) of China. The authors thank Ms Y Deng and D Xiao for technical assistance.

\section{Take home messages}

- Melanoma inhibitor of apoptosis protein (ML-IAP) was expressed in $50-70 \%$ of archived melanoma tissues, depending on the method used, and there was no significant difference between primary and secondary melanomas

- A small number of dermal naevi ( 20\%) also expressed ML-IAP

- Further testing and standardisation are needed for MLIAP to be assayed quantitatively in patients 
Authors' affiliations

J Gong, N Chen, Q Zhou, B Yang, Y Wang, X Wang, Pathology Department, West China Hospital, West China Medical School, Sichuan University, Chengdu, 610041, China

\section{REFERENCES}

1 Soengas MS, Lowe SW. Apoptosis and melanoma chemoresistance. Oncogene 2003;22:3138-51.

2 Vucic D, Stennicke HR, Pisabarro MT, et al. ML-IAP, a novel inhibitor of apoptosis that is preferentially expressed in human melanomas. Curr Biol 2000; 10:1359-66.

3 Kasof GM, Gomes BC. Livin, a novel inhibitor of apoptosis protein family member. J Biol Chem 2001;276:3238-46.

4 Lin JH, Deng G, Huang Q, et al. KIAP, a novel member of the inhibitor of apoptosis protein family. Biochem Biophys Res Commun 2000;279:820-31

5 Ashhab Y, Alian A, Polliack A, et al. Two splicing variants of a new inhibitor of apoptosis gene with different biological properties and tissue distribution pattern. FEBS Lett 2001;495:56-60.

6 Salvesen GS, Duckett CS. IAP proteins: blocking the road to death's door. Nat Rev Mol Cell Biol 2002;3:401-10.

7 Chai J, Du C, Wu JW, et al. Structural and biochemical basis of apoptotic activation by Smac/DIABLO. Nature 2000;406:855-62.

8 Du C, Fang M, Li Y, et al. Smac, a mitochondrial protein that promotes cytochrome c-dependent caspase activation by eliminating IAP inhibition. Cell 2000;102:33-42.

9 Verhagen AM, Ekert PG, Pakusch M, et al. Identification of DIABLO, a mammalian protein that promotes apoptosis by binding to and antagonizing IAP proteins. Cell 2000; 102:43-53.

10 Srinivasula SM, Datta P, Fan XJ, et al. Molecular determinants of the caspasepromoting activity of Smac/DIABLO and its role in the death receptor pathway. J Biol Chem 2000;275:36152-7.

11 Vucic D, Deshayes K, Ackerly H, et al. SMAC negatively regulates the antiapoptotic activity of melanoma inhibitor of apoptosis (ML-IAP). J Biol Chem 2002;277:12275-9

12 Schmollinger JC, Vonderheide RH, Hoar KM, et al. Melanoma inhibitor of apoptosis protein (ML-IAP) is a target for immune-mediated tumour destruction. Proc Natl Acad Sci U S A 2003;100:3398-403.

13 Schmollinger JC, Dranoff G. Targeting melanoma inhibitor of apoptosis protein with cancer immunotherapy. Apoptosis 2004;9:309-13.

14 Andersen MH, Reker S, Becker JC, et al. The melanoma inhibitor of apoptosis protein: a target for spontaneous cytotoxic T cell responses. J Invest Dermatol 2004; 122:392-9
15 Franklin MC, Kadkhodayan S, Ackerly H, et al. Structure and function analysis of peptide antagonists of melanoma inhibitor of apoptosis (ML-IAP). Biochemistry 2003;42:8223-31.

16 Sun H, Nikolovska-Coleska Z, Yang CY, et al. Structure-based design of potent, conformationally constrained Smac mimetics. J Am Chem Soc 2004; 126:16686-7.

17 Komminoth P, Roth J, Schroder S, et al. Overlapping expression of immunohistochemical markers and synaptophysin mRNA in pheochromocytomas and adrenocortical carcinomas. Implications for the differential diagnosis of adrenal gland tumors. Lab Invest 1995:72:424-31.

18 Inagaki H, Okabe M, Seto M, et al. API2-MALT1 fusion transcripts involved in mucosa-associated lymphoid tissue lymphoma: multiplex RT-PCR detection using formalin-fixed paraffin-embedded specimens. Am J Pathol 2001;158:699-706

19 Vucic D, Franklin MC, Wallweber HJ, et al. Engineering ML-IAP to produce an extraordinarily potent caspase 9 inhibitor: implications for Smac-dependent anti-apoptotic activity of ML-IAP. Biochem J 2005;385:1 1-20.

20 Ross JS, Fletcher JA, Bloom KJ, et al. HER-2/neu testing in breast cancer. Am J Clin Pathol 2003;120(suppl):S53-71.

21 Luftner D, Genvresse I, Geppert R, et al. Lack of HER-2/neu overexpression in non-Hodgkin's lymphoma. Anticancer Res 2004;24:3233-7.

22 Nachmias B, Ashhab Y, Bucholtz V, et al. Caspase-mediated cleavage converts livin from an antiapoptotic to a proapoptotic factor: implications for drug-resistant melanoma. Cancer Res 2003:63:6340-9.

23 Rhodes A, Borthwick D, Sykes R, et al. The use of cell line standards to reduce HER-2/neu assay variation in multiple European cancer centers and the potential of automated image analysis to provide for more accurate cut points for predicting clinical response to trastuzumab. Am J Clin Pathol 2004:122:51-60.

24 Chiodino C, Cesinaro AM, Ottani D, et al. Communication: expression of the novel inhibitor of apoptosis survivin in normal and neoplastic skin. J Invest Dermatol 1999;113:415-18.

25 Kanzler MH, Mraz-Gernhard S. Primary cutaneous malignant melanoma and its precursor lesions: diagnostic and therapeutic overview. J Am Acad Dermatol 2001;45:260-76.

26 Piepkorn M, Barnhill RL. Common acquired and atypical (dysplastic) melanocytic naevi. In: Barnhill RL, Piepkorn M, Busam KJ, eds. Pathology of melanocytic naevi and malignant melanoma, 2nd ed. New York: Springer, 2004:51-89.

27 Glinsky GV, Glinsky VV, Ivanova AB, et al. Apoptosis and metastasis: increased apoptosis resistance of metastatic cancer cells is associated with the profound deficiency of apoptosis execution mechanisms. Cancer Lett 1997;115:185-93

28 Soengas MS, Capodieci P, Polsky D, et al. Inactivation of the apoptosis effector Apaf-1 in malignant melanoma. Nature 2001;409:207-11. 